\title{
A quality improvement project on improving the compliance of 'oxygen prescription with target saturations' in a district general hospital
}

\author{
Authors: Piyush Singh, ${ }^{\mathrm{A}}$ May New, ${ }^{\mathrm{A}}$ Aye $\mathrm{Mon}^{\mathrm{A}}$ and Cathy Hay ${ }^{\mathrm{A}}$
}

\section{Introduction}

Giving an appropriate oxygen therapy plays an important role in the management of the patients. Inappropriate and improper use of oxygen can bring risk to the patients including hypercapnia.

The British Thoracic Society (BTS) states that oxygen must be prescribed within desired target saturations of $94-98 \%$ and those at risk of hypercapnia should receive restrictive oxygen with a target saturation of $88-92 \%$. $^{1,2}$

We aimed at improving the rate of oxygen prescription in compliance with the BTS guidelines in a 300-bedded small district general hospital through education, training and staff awareness to improve the quality of healthcare.

\section{Objectives}

The objective was to achieve over $95 \%$ prescription rate of oxygen for patients who are to be given oxygen as part of their treatment during their stay in the hospital.

Oxygen should be prescribed on the drug chart within the target saturations and also the target saturations should be mentioned on the nursing observations chart and signed by the prescriber.

\section{Methods and strategy}

A quality improvement project was undertaken at Furness General Hospital (a district general hospital) across the medical wards over a period of 4 months and studied on two different occasions.

\section{Aim}

The aim was to improve the oxygen prescription rate and to increase awareness about its importance among doctors and nurses.

The initial phase of data collection was done during December 2018 which included all the patients who were given oxygen as a part of their treatment from their admission, and the results were presented in the medical meetings ( $n=34$ (male 21; female 13); mean age 78 years)

Authors: ${ }^{A}$ University Hospitals of Morecambe Bay NHS Foundation Trust, Kendal, UK
We also sent the questionnaires to medical staff which included all grades of doctor to find out about their awareness of prescription of oxygen as a drug. We found out that they are not fully aware of the oxygen prescribing methods as a drug and needed teaching and training about it.

Thereafter, steps were taken to raise awareness among the medical and nursing staff about the importance of oxygen prescribing. This was achieved by displaying posters on the wards and clerking areas, updating the e-learning on the trust intranet, local teaching, sending out e-posters through emails and conducting online surveys.

After implementation, we collected the data again to study the post-intervention results ( $n=30$ (male 16; female 14); mean age 71 years).

\section{Results and discussion}

We were able to achieve the improvement in oxygen prescription with correct target saturations from $14 \%$ in our first cycle to $66 \%$ in our second cycle done in March-April 2019. Although the overall prescription rate improved from $24 \%$ to $95 \%$ (including both online prescription and paper observation charts), the rate of prescription with correct target saturations improved from $14 \%$ to $66 \%$. We were also able to achieve a reduction in the percentage of patients being given high-flow oxygen despite being at risk of hypercapnia from $12 \%$ to $0 \%$.

\section{Conclusion}

The aim of the quality improvement project was to achieve $95 \%$ prescription rate with correct saturations. We achieved to increase the correct prescription rate to $66 \%$ (from 14\%) which was a significant improvement. Although we couldn't achieve the $95 \%$ target, we made a significant improvement to the current practice by simply educating, training and creating awareness. We also made arrangements for future medical teams in the trust to achieve higher targets by updating the mandatory e-learning to create awareness and also through posters. We were also able to identify areas of improvement which could improve future results.

This is in line with previous oxygen prescription audits which had shown improvements after interventions. Areas of improvements 
in future were identified and interventions were planned such as to deal with (either prescribe or delete) a mandatory oxygen prescription form before prescribing regular medications, a mandatory check of prescription during the post-take ward round and also further teachings of medical and nursing staff could improve the results further.

\section{Conflicts of interest}

None declared.

\section{References}

1 O'Driscoll BR, Howard LS, Earis ] et al. British Thoracic Society guideline for oxygen use in adults in healthcare and emergency settings. BMJ Open Respiratory Research 2017:4:e000170. https:// bmjopenrespres.bmj.com/content/4/1/e000170

2. O'Driscoll BR, Howard LS, Earis ] et al. BTS Guideline for oxygen use in adults in healthcare and emergency settings. Thorax 2017; 72:i1-90. www.brit-thoracic.org.uk/document-library/guidelines/ emergency-oxygen/bts-guideline-for-oxygen-use-in-adults-inhealthcare-and-emergency-settings 